\title{
A real-world study of the efficacy and safety of anti-programmed cell death-1 therapy combined with chemotherapy or targeted therapy in patients with advanced biliary tract cancer
}

\author{
Danyang Sun ${ }^{1 \#}$, Junxun Ma ${ }^{2 \#}$, Jinliang Wang', Lijie Wang ${ }^{2}$, Sujie Zhang ${ }^{2}$, Guangying Chen ${ }^{2}$ Xiaoyan Li $^{2}$, \\ Pengfei Cui ${ }^{2}$, Xuan Zheng ${ }^{2}$, Yi $\mathrm{Hu}^{2} \wedge$ \\ ${ }^{1}$ Intensive Care Unit, West Ward, China-Japan Friendship Hospital, Beijing, China; ${ }^{2}$ Department of Medical Oncology, Chinese PLA General \\ Hospital, Beijing, China \\ Contributions: (I) Conception and design: J Ma, Y Hu; (II) Administrative support: J Ma, J Wang; (III) Provision of study materials or patients: D Sun, \\ L Wang; (IV) Collection and assembly of data: D Sun, G Chen, X Li; (V) Data analysis and interpretation: D Sun, P Cui, X Zheng; (VI) Manuscript \\ writing: All authors; (VII) Final approval of manuscript: All authors. \\ "These authors contributed equally to this work as co-first authors. \\ Correspondence to: Prof. Yi Hu. Department of Medical Oncology, Chinese PLA General Hospital, 28 Fuxing Road, Haidian, Beijing 100853, China. \\ Email: huyi301zlxb@sina.com.
}

Background: Immune checkpoint inhibitors (ICIs) represent a breakthrough in cancer treatment. However, they have rarely been used to treat biliary tract cancer (BTC). In the current study, we aimed to evaluate and compare the efficacy and safety of anti-programmed cell death-1 (PD-1) therapy used alone or in combination with chemotherapy or targeted therapy in the treatment of advanced BTC.

Methods: Patients with advanced BTC who were treated either with anti-PD-1 therapy alone or antiPD-1 therapy plus chemotherapy or targeted therapy between December, 2015 and October, 2017 were retrospectively screened for eligibility. Patients who had previously received treatment with any agent targeting T-cell co-stimulation or immune checkpoints were excluded. Overall survival (OS), progressionfree survival (PFS), objective response rate (ORR), and safety were evaluated.

Results: A total of 37 patients were included in this study (15 cases in the monotherapy group and 22 cases in the combination group). Patients in the combination group had significantly longer OS [median, 8.2 vs. 3.6 months, HR 0.47 (0.20-1.10), $\mathrm{P}=0.011$ ] and PFS (median, 3.9 vs. 2.0 months, HR 0.58 (0.28-1.19), $\mathrm{P}=0.034)$ than patients in the monotherapy group. The ORR was $18.2 \%(4 / 22)$ and $0 \%$ in the combination group and monotherapy group, respectively, and the difference was not significant $(\mathrm{P}=0.131)$. Furthermore, no significant difference was found between the two groups with respect to the incidence of grade 3-4 treatment-related adverse events $(\mathrm{P}=0.388)$.

Conclusions: Anti-PD-1 therapy plus chemotherapy or targeted therapy is an effective and tolerable treatment for patients with advanced BTC and is promising as a first-line treatment or beyond.

Keywords: Biliary tract cancer (BTC); programmed cell death-1 inhibitors (PD-1 inhibitors); combination; efficacy; safety

Submitted Sep 17, 2020. Accepted for publication Dec 18, 2020.

doi: 10.21037/jgo-20-562

View this article at: http://dx.doi.org/10.21037/jgo-20-562

^ ORCID: Yi Hu, 0000-0001-9319-5692. 


\section{Introduction}

Biliary tract cancer (BTC) is an invasive heterogeneous malignant tumor. The three major types of BTC are intrahepatic cholangiocarcinoma (ICC), extrahepatic cholangiocarcinoma (ECC), and gallbladder carcinoma (GBC) (1). BTC has a high mortality rate, and while it is rare in Western countries, it is common in East Asia $(2,3)$. Surgery is the only curative treatment for BTC; however, approximately $90 \%$ of BTC cases are unresectable. For patients with unresectable or metastatic BTC, the recommended standard therapy is conventional chemotherapy, such as gemcitabine plus platinum. Unfortunately, however, the efficacy of chemotherapy is far from ideal. The objective response rate (ORR) is rather low (4), the median overall survival (OS) of BTC ranges from 6-8 months, and the 5-year survival rate is less than $10 \%(5-7)$. Therefore, there is an urgent need for more effective strategies for the treatment of advanced BTC.

Immune checkpoint inhibitors (ICIs), which utilize tumor-targeted monoclonal antibodies, have emerged as a promising treatment option for multiple malignancies, and especially for chemo-resistant solid tumors such as renal cell carcinoma (8). Although the immune system plays a key role in the etiology of BTC, limited evidence exists on the activity of checkpoint inhibitors in this disease. Pembrolizumab has been approved by the United States Food and Drug Administration (FDA) for the treatment of microsatellite instability-high (MSI-H) or mismatch repair-deficient (dMMR) tumors. A phase II clinical trial showed that $\mathrm{dMMR}$ patients who received pembrolizumab had a higher ORR and longer progression-free survival (PFS) than MMR-proficient patients. Among these dMMR patients, the four included dMMR BTC patients had an ORR of $50 \%$ (9). However, dMMR/MSI-H mutations occur infrequently in BTC, with the frequency of MSI-H reported as being less than $10 \%$ for different BTC subtypes (10). Data from the KEYNOTE-028 clinical trial showed that antiprogrammed cell death-1 (PD-1) monotherapy achieved an ORR of $17 \%$ when used as a second-line or beyond treatment in programmed death-ligand 1 (PD-L1)-positive pretreated BTC patients (11). An observational study of ICIs combined with lenvatinib in patients with pretreated advanced intrahepatic cholangiocarcinoma (ICC) reported an ORR of $21.4 \%(3 / 14)$, a disease control rate (DCR) of $92.9 \%(13 / 14)$, and a median PFS of 5.9 months (12). Based on these limited results, ICIs seemingly have modest efficacy in treating BTC.
An increasing amount of evidence suggests that chemotherapy in combination with immunotherapy may induce beneficial changes in both the immune system and the tumor to enhance antitumor immunity. Gemcitabine and cisplatin have been shown to increase the expression of human leukocyte antigen on tumor cells (13) and to decrease that of programmed death-ligand 2 (PD-L2) on tumor cells, respectively (14). Besides, targeted therapy, which was originally developed to decrease tumor viability in a cell-autonomous manner, may also modulate immune cell functions and combine with immune-based therapies to increase the durable clinical response (15). Thus, the efficacy and safety of anti-PD-1 monotherapy and antiPD-1 therapy plus chemotherapy or targeted therapy in the treatment of advanced BTC need to be further evaluated.

In the present study, we evaluated and compared the efficacy and safety of anti-PD-1 monotherapy and antiPD-1 therapy plus chemotherapy or targeted therapy as the first-line treatment or beyond for patients with advanced BTC. This study presents a new treatment option for a disease that currently has a dismal prognosis and few effective treatments.

We present the following article in accordance with the STROBE reporting checklist (available at http://dx.doi. org/10.21037/jgo-20-562).

\section{Methods}

\section{Study design and participants}

Patients with advanced BTC who received at least one dose of anti-PD-1 therapy at the Chinese People's Liberation Army (PLA) General Hospital of the between December, 2015 and October, 2017 were enrolled in this retrospective study. To ensure the quality of data, the study protocol, case report form (CRF), and the standard operating specification (SOP) of data collection were prospectively designed before the commencement of this study. The study was approved by Chinese PLA General Hospital's review board (No. S2018-144-02) and written informed consent was obtained from all patients. All procedures performed in this study involving human participants were in accordance with the Declaration of Helsinki (as revised in 2013).

Enrolled patients met the following eligibility criteria: (I) histologically proven metastatic BTC; (II) received at least one dose of ICI monotherapy or ICI-based combination therapy. Patients had previously received any agent targeting T-cell co-stimulation or immune checkpoints 
were excluded.

\section{Data collection and study objectives}

Two researchers independently extracted and verified the data of the patients, including information on their clinicopathological characteristics and treatment history. The imaging data were assessed by two physicians independently. Any inconsistencies in the evaluation results were resolved by the director of the imaging center. The last data were collected on February 28, 2018. The primary study objective was OS. The secondary objectives were PFS, ORR, DCR by Response Evaluation Criteria in Solid Tumors (RECIST) version 1.1 (16), and safety (adverse events). Adverse events were defined according to the National Cancer Institute Common Terminology Criteria for Adverse Events, version 4.0 (17). Patients without recorded clinical or radiographic disease progression or death were censored on the date of the last visit. We report this study according to the Transparent Reporting of Evaluations with Nonrandomized Designs (TREND) reporting guideline (18).

\section{Statistical analysis}

Baseline characteristics and response data between the two groups were compared using the chi-squared test or Fisher's exact test for categorical variables and the MannWhitney $U$ test for continuous or ordinal variables. Survival analysis was performed using Kaplan-Meier curves, with a $\mathrm{P}$ value determined with the Breslow test. Hazard ratios were estimated through Cox proportional hazards regression. For multivariable analyses, variables with $\mathrm{P} \leq 0.05$ in univariable analysis or were entered into the multivariable model. A two-sided $\mathrm{P}$ value of $<0.05$ was considered statistically significant. All statistical analyses were performed with SPSS statistical software (version 20.0, IBM Corporation, USA).

\section{Results}

\section{Patient characteristics and treatment}

Between December, 2015 and October, 2017, 37 patients accepted at least one dose of immunotherapy as a firstline or later treatment; of them, 15 patients received antiPD-1 monotherapy (the monotherapy group) and 22 patients were treated with anti-PD-1 combination therapy (the combination group) (Figure 1). In the combination group, three (13.6\%) patients received anti-PD-1 therapy plus targeted therapy (nimotuzumab or apatinib), while the rest $(86.4 \%)$ received a combination of anti-PD-1 therapy and chemotherapy. The individual treatment strategies are shown in Table S1. The groups were well balanced in terms of demographics and disease characteristics (Table 1). The majority of patients in both groups had cholangiocarcinoma. The percentage of never-smokers was $72.7 \%$ and $60 \%$ in the combination group and the monotherapy group, respectively. A higher Eastern Cooperative Oncology Group (ECOG) status score, no viral infection, and a low histological grade were the dominant features of good prognosis. Two-thirds of the patients in both groups had undergone surgical treatment, and nearly half of the patients had received systemic treatment. The liver was the most common metastatic site. Seventy-eight percent of all patients received nivolumab alone or a nivolumab-based treatment regimen.

\section{Efficacy}

In total, 31 (83.8\%) progression events and 23 (62.2\%) deaths were recorded. The median OS was 8.2 months (95\% CI: 4.01-12.39 months) in combination therapy group compared with 3.6 months (95\% CI: 0.73-6.47 months) in the monotherapy group (HR 0.47, 95\% CI: 0.20-1.10, $\mathrm{P}=0.011$, Figure $2 A$ ). When body mass index (BMI), ECOG status, number of metastases, and number of prior lines for metastatic disease were included in the multivariable regression, the adjusted HR was 0.34 (95\% CI: 0.11-1.06). The median PFS was 3.9 months (95\% CI: 2.53-5.27 months) with combination therapy and 2.0 months (95\% CI: $1.12-2.88$ months) with monotherapy (HR 0.58, 95\% CI: $0.28-1.19, \mathrm{P}=0.034$, Figure $2 B$ ). The adjusted HR was 0.68 (95\% CI: 0.31-1.52) when ECOG status and $\mathrm{BMI}$ were included in the multivariable regression. The results of subgroup analyses of OS and PFS are shown in Figure S1. The ORR was higher in the combination group than in the monotherapy group, but the difference was not significant $(18.2 \%$ vs. $0 \%, \mathrm{P}=0.131)$, and similar results were observed in the DCR $(83.4 \%$ vs. $66.7 \%, \mathrm{P}=0.228$, Table 2). In regard to tumor shrinkage, $54.5 \%(12 / 22)$ of patients in the combination group and $40 \%(6 / 15)$ of patients in the monotherapy group achieved a decrease from baseline in the sum of the target lesions (Figure 3). The median change from baseline was $-3.5 \%(-100 \%$ to $33 \%)$ in the combination group and $5 \%(-18 \%$ to $48 \%)$ in 


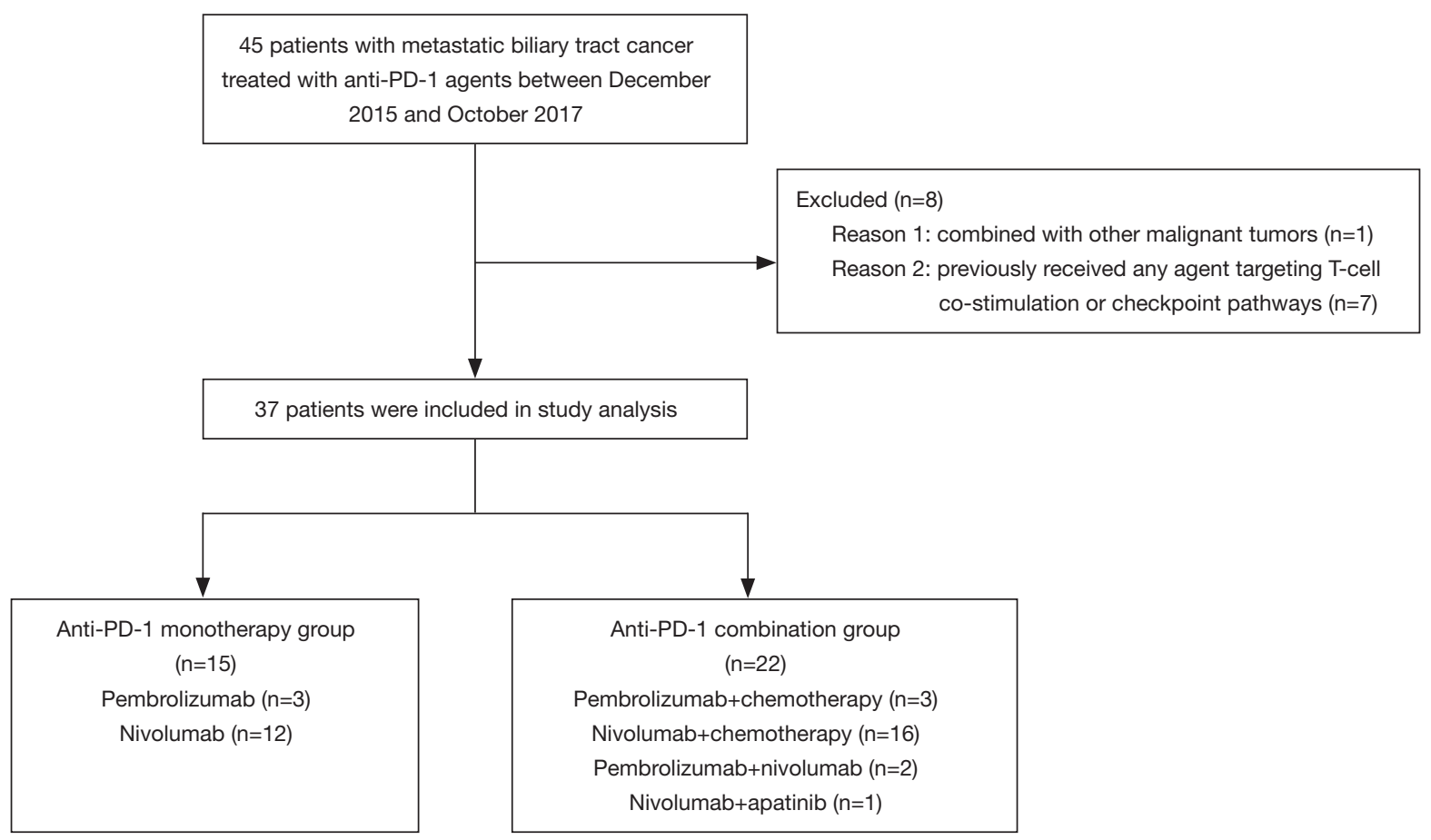

Figure 1 Flow diagram of the study.

Table 1 Baseline characteristics

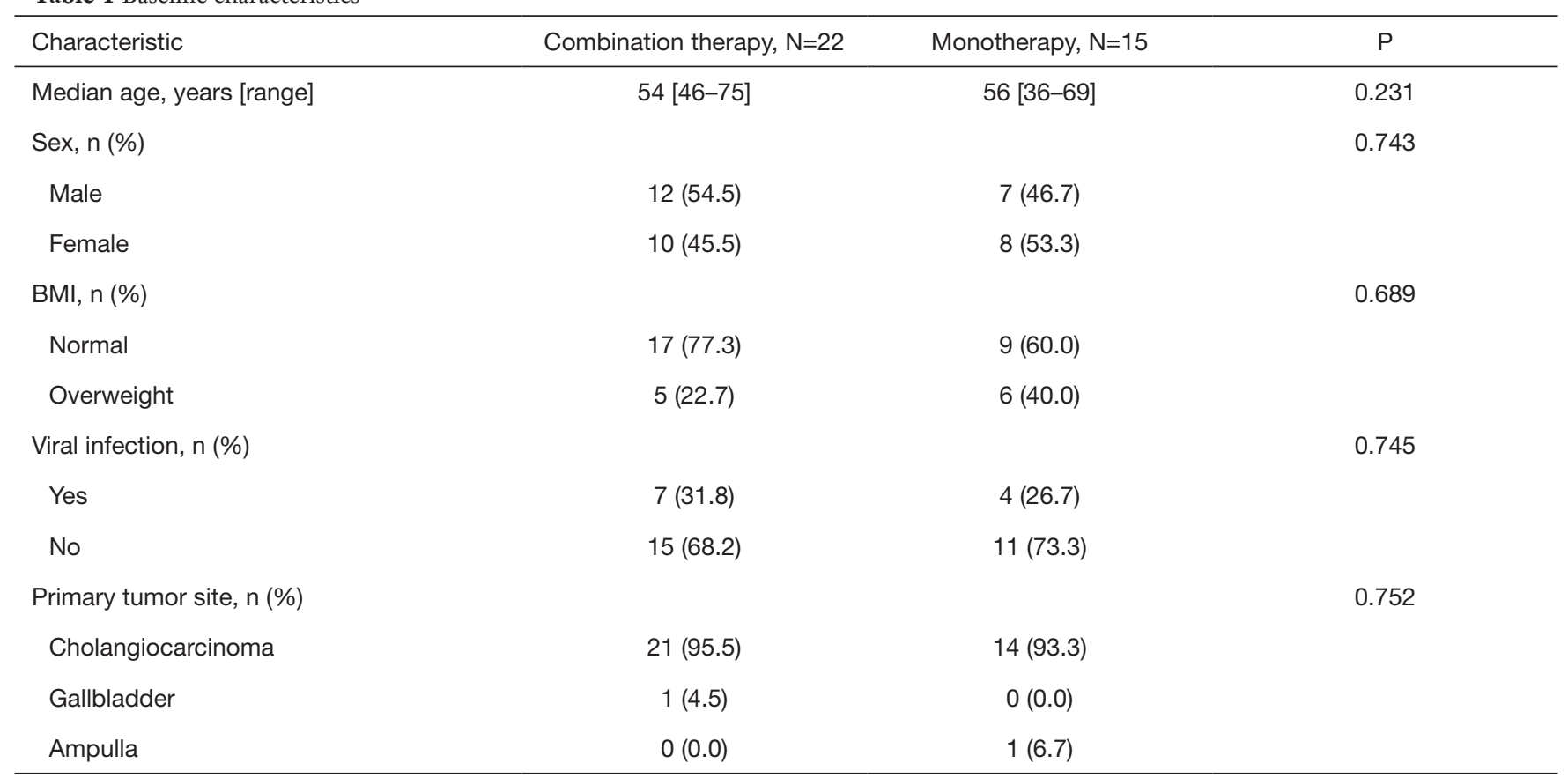

Table 1 (continued) 
Table 1 (continued)

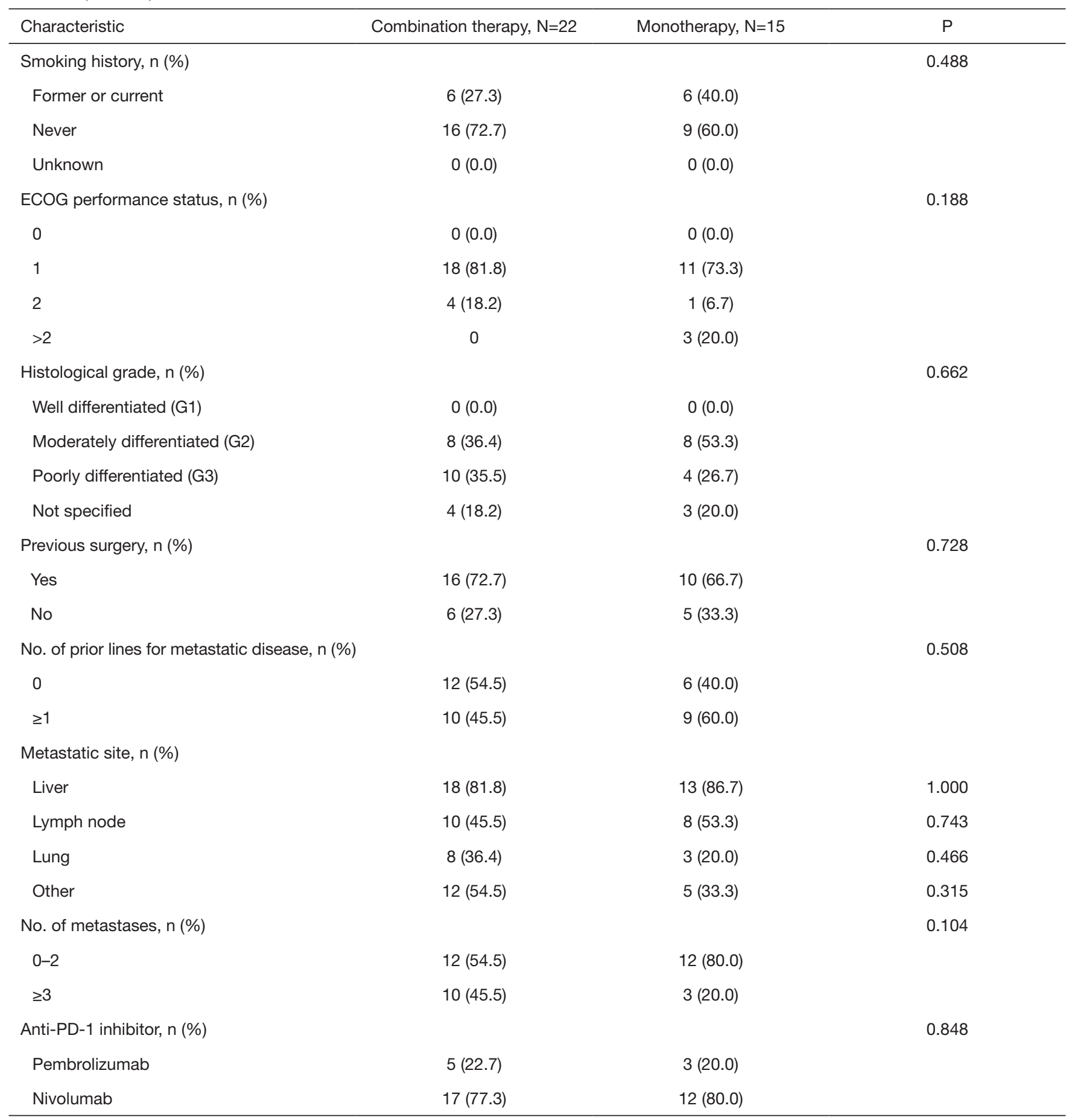

In 2002, based on the data of each province of China, China Working Group on Obesity (WGOC) suggested classifying a BMI between $24.0-28.0 \mathrm{~kg} / \mathrm{m}^{2}$ as overweight and $\geq 28.0 \mathrm{~kg} / \mathrm{m}^{2}$ as obese among Chinese populations (19). PD-1, programmed cell death-1; ECOG, Eastern Cooperative Oncology Group; BMI, body mass index. 
the monotherapy group.

\section{Safety}

Treatment-related adverse events (TRAEs) observed across all grades are summarized in Table 3. In the combination group and monotherapy group, TRAEs of any grade occurred in $59.1 \%$ and $20.0 \%$ of patients,
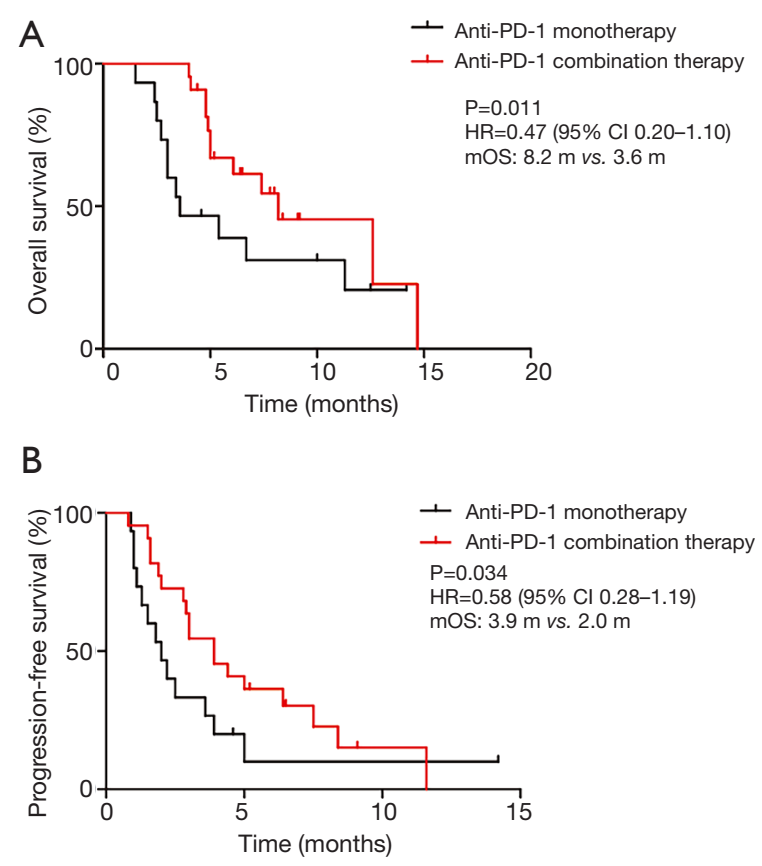

Figure 2 Kaplan-Meier estimates for overall survival (OS) and progression-free survival (PFS). (A) OS between the monotherapy and combination therapy groups. (B) PFS between the monotherapy and combination therapy groups. respectively $(\mathrm{P}=0.040)$. The most common TRAEs with combination treatment were leukopenia $(9 / 22,40.9 \%)$, thrombocytopenia $(5 / 22,22.7 \%)$, and nausea $(4 / 22$, $18.2 \%)$, while fatigue $(2 / 15,13.3 \%)$ and increased aspartate aminotransferase $(2 / 15,13.3 \%)$ were the most common monotherapy-associated adverse events.

There was no significant difference between the two groups with respect to the incidence of grade 3-4 toxic effects $(18.2 \%$ vs. $6.7 \%, \mathrm{P}=0.388)$. The most commonly reported grade $\geq 3$ events were thrombocytopenia [3 (13.6\%) patients], leukopenia [2 (9.1\%) patients], and alopecia [1 $(4.5 \%)$ patient] in the combination group, and thrombocytopenia [1 $(6.7 \%)$ patient] in monotherapy group. No drug-related deaths were reported in either group.

\section{Discussion}

The present retrospective study evaluated the efficacy and safety of anti-PD-1 therapy alone or in combination with chemotherapy or targeted therapy as a first-line treatment or beyond for advanced BTC patients. We found that the combination group achieved significantly longer OS and PFS than the anti-PD-1 monotherapy group, and also showed an increased ORR and DCR, albeit without significant difference. Similar results were observed in most subgroups. Although TRAEs of any grade occurred more frequently in the combination group than in the monotherapy group, no significant difference existed in the incidence of grade 3-4 toxic effects between the two groups. These results provide more evidence that anti-PD-1 combination therapy is effective and tolerable for advanced BTC.

Treatment-naïve $(\mathrm{n}=18)$ and pretreated $(\mathrm{n}=19)$ patient

Table 2 Tumor response to treatment for overall cohort

\begin{tabular}{lcc}
\hline Variable & Monotherapy, $\mathrm{N}=15$ & Combination therapy, N=22 \\
\hline Objective response, $\mathrm{n}(\% ; 95 \% \mathrm{Cl})$ & 0 & $4(18.2 ; 6.5-36.9)$ \\
Disease control rate, $\mathrm{n}(\% ; 95 \% \mathrm{Cl})$ & $10(66.7 ; 42.2-58.8)$ & $19(83.4 ; 68.4-96.2)$ \\
Best overall response, $\mathrm{n}(\%)$ & 0 & $1(4.5)$ \\
Complete response & 0 & $3(13.6)$ \\
Partial response & $10(66.7)$ & $15(68.2)$ \\
Stable disease & $5(33.3)$ & $3(13.6)$ \\
Progressive disease & & 0.228 \\
\hline
\end{tabular}

PD-1, programmed cell death-1. 
A

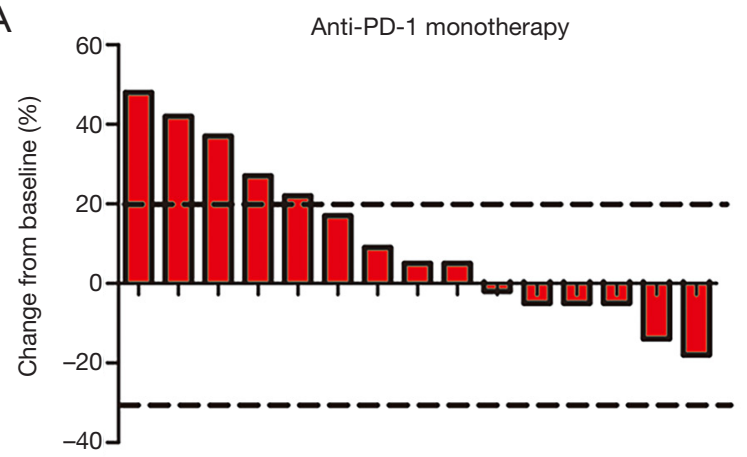

B

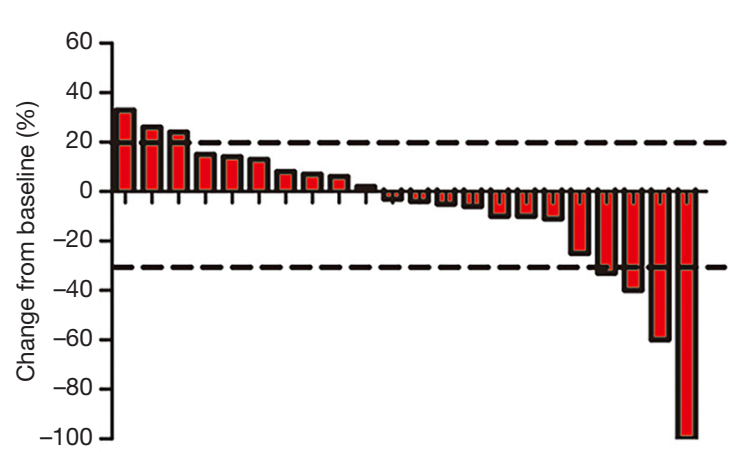

Figure 3 Waterfall plots of best percentage change in tumor size. (A) The best percentage change from baseline in tumor size for individual patient in the monotherapy group. (B) The best percentage change from baseline in tumor size for individual patient in the combination therapy group.

subpopulations were also evaluated in this study. For therapy-naive patients, the median OS and PFS with antiPD-1 monotherapy (3.7 and 1.7 months, respectively) were found to be similar to those with conventional therapy such as gemcitabine based chemotherapy $(20,21)$. The addition of chemotherapy or targeted therapy prolonged OS and PFS (12.8 and 4.0 months, respectively; Figure S2). For the patients who had previously received systemic therapy, our results showed a slightly lower ORR, DCR, and median PFS in the combination group $(0 \%, 90 \%$, and 3.5 months, respectively; Table S2 and Figure S3) than those reported by a previous study on the combination treatment of ICIs and lenvatinib in patients with pretreated advanced ICC (12). The possible reasons for this difference might be that in our study, the majority of the patients accepted ICIs plus chemotherapy, and the pathological type was more complex.

Systemic chemotherapy is the mainstay of treatment for advanced BTC (22). Although chemotherapy is a major method that attack tumor cells, cumulative evidence indicates that cytotoxic drugs also affect the immune system by increasing the ratio of cytotoxic lymphocytes to regulatory $\mathrm{T}$ cells and inhibiting myeloid-derived suppressor cells, leading to tumor regression $(23,24)$. The KEYNOTE-189 clinical trial showed that patients with advanced non-small-cell lung cancer (NSCLC) who received pembrolizumab combined with chemotherapy had a significantly longer OS and PFS (25). A phase Ib/II clinical trial to evaluate the efficacy of pembrolizumab combined with gemcitabine and nab-paclitaxel in the treatment of pancreatic cancer showed that this combination achieved a DCR of $100 \%$ and had acceptable toxicity (26). These findings support ICIs plus chemotherapy as a potential rational strategy for cancer treatment. In the current study, $86.4 \%$ of patients $(n=19)$ in the combination group received anti-PD-1 therapy combined with chemotherapy, and the ORR and DCR was $21.1 \%$ and $84.2 \%$, respectively. This result indicated that the combination treatment might improve the therapeutic response in BTC, although further validation is still needed.

Insights from several studies have provided a framework for the combination of molecular targeted inhibitors and anti-PD-1 therapy. Mutations of epidermal growth factor receptor (EGFR) which commonly occur in BTC, constitutively drive the mitogen-activated protein kinase (MAPK) pathway to promote tumor cell proliferation and survival (27). Basic research has revealed that inhibition of the MAPK pathway can enhance host antitumor immunity via multiple mechanisms, including by improving $\mathrm{T}$ cell function and elevating antigen expression, to regulate the tumor microenvironment for enhanced immune response (28). The combination of atezolizumab and erlotinib has been shown to be effective, with an ORR of $75 \%$ and a manageable safety profile (29). A study of the combination of tremelimumab with gefitinib in EGFRmutant NSCLC reported a DCR of $62 \%$ and a safety profile that was in line with that previously described (30). Previous studies demonstrated that the combination of ICIs with vascular endothelial growth factor A (VEGF-A) or vascular endothelial growth factor receptor (VEGFR) inhibitors enhanced tumor immune responses. A study of ipilimumab plus bevacizumab therapy $v s$. ipilimumab monotherapy in the treatment of metastatic melanoma indicated that the combination therapy resulted in increased adhesion molecule expression and increased intratumoral 
Table 3 Treatment-related adverse events

\begin{tabular}{|c|c|c|c|c|}
\hline Adverse event & \multicolumn{2}{|c|}{ ICI monotherapy $(\mathrm{N}=15)$} & \multicolumn{2}{|c|}{$\mathrm{ICI}$ combination therapy $(\mathrm{N}=22)$} \\
\hline Any term & $3(20.0 \%)$ & $1(6.7 \%)$ & $13(59.1 \%)$ & $4(18.2 \%)$ \\
\hline Nausea & $1(6.7 \%)$ & 0 & $4(18.2 \%)$ & 0 \\
\hline Diarrhea & $1(6.7 \%)$ & 0 & 0 & 0 \\
\hline Anemia & 0 & 0 & $2(9.1 \%)$ & 0 \\
\hline Alopecia & 0 & 0 & $1(4.5 \%)$ & $1(4.5 \%)$ \\
\hline Skin rash & 0 & 0 & $1(4.5 \%)$ & 0 \\
\hline Vomiting & 0 & 0 & $1(4.5 \%)$ & 0 \\
\hline Thrombocytopenia & $1(6.7 \%)$ & $1(6.7 \%)$ & $5(22.7 \%)$ & $3(13.6 \%)$ \\
\hline Leukopenia & 0 & 0 & $9(40.9 \%)$ & $2(9.1 \%)$ \\
\hline
\end{tabular}

$\mathrm{ICl}$, immune checkpoint inhibitor.

immune cell infiltration (31). Several studies have also reported a promising ORR for ICIs plus VEGF pathway inhibitors such as sunitinib, pazopanib, and bevacizumab in some malignant tumors $(32,33)$. In our study, three patients in the combination group received ICIs plus molecular targeted inhibitors (EGFR monoclonal antibody nimotuzumab, $\mathrm{n}=2$; angiogenesis inhibitor apatinib, $\mathrm{n}=1$ ) and achieved a DCR of $100 \%$. However, the sample size was small, and the efficacy of ICIs combined with targeted inhibitors in BTC needs to be further confirmed in studies with larger cohorts.

The current study has some limitations. Firstly, this was a retrospective study, which might limit the interpretation of the results. Secondly, the small sample size yielded unavoidable selection bias and recall bias. Thirdly, chemotherapy is still the standard treatment for BTC. In the current study, we merely compared ICI-based combination treatment and ICI monotherapy. The clinical responses observed in our patients may have been attributable to chemotherapy or targeted therapy rather than the combined treatment strategy. Although these factors weaken the validity and reliability of our conclusions to some degree, the real-world data could inform a prospective study in the future.

To conclude, anti-PD-1 inhibitor therapy could potentially be a viable treatment option for advanced BTC patients as the first-line therapy or beyond. In particular, the combination of anti-PD-1 therapy and chemotherapy or targeted therapy was found to achieve longer OS and improved PFS and ORR compared to anti-PD-1 monotherapy alone. These clinical results provide valuable clues and pave the way for future prospective studies on BTC.

\section{Acknowledgments}

We thank all staff at the hospital for their contributions to this study.

Funding: This work was supported by the National Natural Science Foundation of China (81402552 to YH, 81672996 to $\mathrm{YH})$.

\section{Footnote}

Reporting Checklist: The authors have completed the STROBE reporting checklist. Available at http://dx.doi. org/10.21037/jgo-20-562

Data sharing statement: Available at http://dx.doi. org/10.21037/jgo-20-562

Conflicts of Interest: All authors have completed the ICMJE 
uniform disclosure form (available at http://dx.doi. org/10.21037/jgo-20-562). The authors have no conflicts of interest to declare.

Ethical Statement: The authors are accountable for all aspects of the work in ensuring that questions related to the accuracy or integrity of any part of the work are appropriately investigated and resolved. The study was approved by the institutional review board of People's Liberation Army General Hospital, Beijing, China (No. S2018-144-02) and written informed consent was obtained from all patients. All procedures performed in this study involving human participants were in accordance with the Declaration of Helsinki (as revised in 2013).

Open Access Statement: This is an Open Access article distributed in accordance with the Creative Commons Attribution-NonCommercial-NoDerivs 4.0 International License (CC BY-NC-ND 4.0), which permits the noncommercial replication and distribution of the article with the strict proviso that no changes or edits are made and the original work is properly cited (including links to both the formal publication through the relevant DOI and the license). See: https://creativecommons.org/licenses/by-nc-nd/4.0/.

\section{References}

1. de Groen PC, Gores GJ, LaRusso NF, et al. Biliary tract cancers. N Engl J Med 1999;341:1368-78.

2. Randi G, Malvezzi M, Levi F, et al. Epidemiology of biliary tract cancers: an update. Ann Oncol 2009;20:146-59.

3. Waisberg DR, Pinheiro RS, Nacif LS, et al. Resection for intrahepatic cholangiocellular cancer: new advances. Transl Gastroenterol Hepatol 2018;3:60.

4. Malka D, Cervera P, Foulon S, et al. Gemcitabine and oxaliplatin with or without cetuximab in advanced biliarytract cancer (BINGO): a randomised, open-label, noncomparative phase 2 trial. Lancet Oncol 2014;15:819-28.

5. Charbel H, Al-Kawas FH. Cholangiocarcinoma: epidemiology, risk factors, pathogenesis, and diagnosis. Curr Gastroenterol Rep 2011;13:182-7.

6. DeOliveira ML, Cunningham SC, Cameron JL, et al. Cholangiocarcinoma: thirty-one-year experience with 564 patients at a single institution. Ann Surg 2007;245:755-62.

7. Mondaca S, Nervi B, Pinto M, et al. Biliary tract cancer prognostic and predictive genomics. Chin Clin Oncol 2019;8:42.
8. Motzer RJ, Escudier B, McDermott DF, et al. Nivolumab versus Everolimus in Advanced Renal-Cell Carcinoma. N Engl J Med 2015;373:1803-13.

9. Le DT, Uram JN, Wang H, et al. PD-1 blockade in tumors with mismatch-repair deficiency. N Engl J Med 2015;372:2509-20.

10. Silva VW, Askan G, Daniel TD, et al. Biliary carcinomas: pathology and the role of DNA mismatch repair deficiency. Chin Clin Oncol 2016;5:62.

11. Bang YJ, Doi T, Braud FD, et al. Safety and efficacy of pembrolizumab (MK-3475) in patients (pts) with advanced biliary tract cancer: Interim results of KEYNOTE-028. Eur J Cancer 2015;51:S112.

12. Lin JZ, Shi WW, Zhao SH, et al. Lenvatinib plus checkpoint inhibitors in patients (pts) with advanced intrahepatic cholangiocarcinoma (ICC): Preliminary data and correlation with next-generation sequencing. J Clin Oncol 2018;36:S500.

13. Liu WM, Fowler DW, Smith P, et al. Pretreatment with chemotherapy can enhance the antigenicity and immunogenicity of tumours by promoting adaptive immune responses. Br J Cancer 2010;102:115-23.

14. Lesterhuis WJ, Punt CJ, Hato SV, et al. Platinum-based drugs disrupt STAT6-mediated suppression of immune responses against cancer in humans and mice. J Clin Invest 2011;121:3100-8.

15. Gotwals P, Cameron S, Cipolletta D, et al. Prospects for combining targeted and conventional cancer therapy with immunotherapy. Nat Rev Cancer 2017;17:286-301.

16. Eisenhauer EA, Therasse P, Bogaerts J, et al. New response evaluation criteria in solid tumours: revised RECIST guideline (version 1.1). Eur J Cancer 2009;45:228-47.

17. Chen AP, BSN AS, Anadkat MJ, et al. Grading dermatologic adverse events of cancer treatments: The Common Terminology Criteria for Adverse Events Version 4.0. J AM Acad Dermatol 2012;67:1025-39.

18. Des Jarlais DC LC, Crepaz N, TREND Group. Improving the reporting quality of nonrandomized evaluations of behavioral and public health interventions: the TREND statement. Am J Public Health 2004;94:361-6.

19. Zhou B. Predictive values of body mass index and waist circumference to risk factors of related diseases in Chinese adult population. Zhonghua Liu Xing Bing Xue Za Zhi 2002;23:5-10.

20. Agarwal R, Sendilnathan A, Siddiqi NI, et al. Advanced biliary tract cancer: clinical outcomes with $\mathrm{ABC}-02$ regimen and analysis of prognostic factors in a tertiary 
care center in the United States. J Gastrointest Oncol 2016;7:996-1003.

21. Valle JW, Wasan H, Johnson P, et al. Gemcitabine alone or in combination with cisplatin in patients with advanced or metastatic cholangiocarcinomas or other biliary tract tumours: a multicentre randomised phase II study - The UK ABC-01 Study. Br J Cancer 2009;101:621-7.

22. Jordan E, Abou-Alfa GK, Lowery MA. Systemic therapy for biliary cancers. Chin Clin Oncol 2016;5:65.

23. Wang Z, Till B, Gao Q. Chemotherapeutic agentmediated elimination of myeloid-derived suppressor cells. Oncoimmunology 2017;6:e1331807.

24. Roselli M, Cereda V, di Bari MG, et al. Effects of conventional therapeutic interventions on the number and function of regulatory $\mathrm{T}$ cells. Oncoimmunology 2013;2:e27025.

25. Gandhi L, Rodríguez-Abreu D, Gadgeel S, et al. Pembrolizumab plus Chemotherapy in Metastatic NonSmall-Cell Lung Cancer. N Engl J Med 2018;378:2078-92.

26. Weiss GJ, Blaydorn L, Beck J, et al. Phase Ib/II study of gemcitabine, nab-paclitaxel, and pembrolizumab in metastatic pancreatic adenocarcinoma. Invest New Drugs 2018;36:96-102.

27. Wee P, Wang Z. Epidermal Growth Factor Receptor Cell Proliferation Signaling Pathways. Cancers (Basel)

Cite this article as: Sun D, Ma J, Wang J, Wang L, Zhang S, Chen G, Li X, Cui P, Zheng X, Hu Y. A real-world study of the efficacy and safety of anti-programmed cell death-1 therapy combined with chemotherapy or targeted therapy in patients with advanced biliary tract cancer. J Gastrointest Oncol 2020;11(6):1421-1430. doi: 10.21037/jgo-20-562
2017;9:E52.

28. Hughes PE, Caenepeel S, Wu LC. Targeted Therapy and Checkpoint Immunotherapy Combinations for the Treatment of Cancer. Trends Immunol 2016;37:462-76.

29. Ma BBY, Rudin CM, Cervantes A, et al. Preliminary safety and clinical activity of erlotinib plus atezolizumab from a phase Ib study in advanced NSCLC. Annoncol 2016;27:S4410.

30. Planchard D, Barlesi F, Gomez-Roca C, et al. Phase I, safety, tolerability and preliminary efficacy study of Tremelimumab (Trem) in combination with Gefitinib (Gef) in EGFR-mutant (EGFR-mut) NSCLC (GEFTREM). Ann Oncol 2016;27:S1245.

31. Hodi FS, Lawrence D, Lezcano C, et al. Bevacizumab plus ipilimumab in patients with metastatic melanoma. Cancer Immunol Res 2014;2:632-42.

32. Amin A, Plimack ER, Infante JR, et al. Nivolumab (antiPD1; BMS-936558, ONO-4538) in combination with sunitinib or pazopanib in patients (pts) with metastatic renal cell carcinoma (mRCC). J Clin Oncol 2014;32:5010.

33. Lieu C, Bendell J, Powderly JD, et al. Safety and efficacy of MPDL3280A (anti-PDL1) in combination with bevacizumab (BEV) and/or chemotherapy (chemo) in patients (PTS) with locally advanced or meta-static solid tumors. Ann Oncol 2014;25:361. 


\section{Supplementary}

Table S1 Treatment received by patients in the anti-PD-1 monotherapy and combination group.

\begin{tabular}{|c|c|}
\hline Subject ID & Monotherapy group \\
\hline Y1809862 & Pembrolizumab \\
\hline Y2317141 & Nivolumab \\
\hline K0011783 & Nivolumab \\
\hline Y2310621 & Nivolumab \\
\hline Y0088543 & Nivolumab \\
\hline Y1095342 & Pembrolizumab \\
\hline Y2074076 & Nivolumab \\
\hline F129459 & Nivolumab \\
\hline Y1004233 & Nivolumab \\
\hline Y1820335 & Pembrolizumab \\
\hline K0035132 & Nivolumab \\
\hline Y2912819 & Nivolumab \\
\hline F154096 & Nivolumab \\
\hline Y1809743 & Nivolumab \\
\hline Y1095770 & Nivolumab \\
\hline Subject ID & Combination therapy group \\
\hline Y1503747 & Pembrolizumab + nimotuzumab \\
\hline Y1986753 & Nivolumab + gemcitabine \\
\hline Y2340415 & Nivolumab + paclitaxel-albumin \\
\hline Y1325273 & Pembrolizumab + paclitaxel-albumin \\
\hline Y2321289 & Nivolumab + oxaliplatin + tegafur \\
\hline F976637 & Nivolumab + apatinib \\
\hline A159858 & Nivolumab + paclitaxel-albumin $+S-1$ \\
\hline F283855 & Nivolumab + gemcitabine \\
\hline Y1355534 & Nivolumab + S-1 \\
\hline Y1490847 & Nivolumab + gemcitabine + cisplatin \\
\hline A284153 & Nivolumab + gemcitabine + cisplatin \\
\hline Y2522731 & Nivolumab + gemcitabine + cisplatin \\
\hline Y2946275 & Nivolumab + paclitaxel-albumin \\
\hline F669590 & Nivolumab + gemcitabine + cisplatin \\
\hline F971599 & Pembrolizumab + docetaxel \\
\hline Y2998674 & Pembrolizumab + S-1 \\
\hline Y1770934 & Nivolumab + S-1 \\
\hline Y2892524 & Nivolumab + gemcitabine + cisplatin \\
\hline K0041892 & Pembrolizumab + nimotuzumab \\
\hline Y0078386 & Nivolumab + gemcitabine + cisplatin \\
\hline Y2897368 & Nivolumab + gemcitabine + cisplatin \\
\hline Y1703986 & Nivolumab + S-1 \\
\hline
\end{tabular}

(c) Journal of Gastrointestinal Oncology. All rights reserved. 
Table S2 Tumor response to treatment for first-line treatment or beyond

\begin{tabular}{|c|c|c|c|c|}
\hline & \multicolumn{2}{|c|}{ ORR } & \multicolumn{2}{|c|}{ DCR } \\
\hline Anti-PD-1 monotherapy group & 0 & 0 & $4(66.7 \%)$ & $6(66.7 \%)$ \\
\hline Anti-PD-1 combination group & $4(33.3 \%)$ & 0 & $10(83.3 \%)$ & $9(90 \%)$ \\
\hline$P$ value & 0.245 & - & 0.638 & 0.303 \\
\hline
\end{tabular}

Note: a, PD-1, programmed death protein 1b, ORR, Objective response rate; c, DCR, Disease control rate. 
A

\begin{tabular}{|c|c|c|c|c|c|c|c|}
\hline Subgroup & $\begin{array}{l}\text { No. of } \\
\text { Patients }\end{array}$ & $\begin{array}{l}\text { Anti-PD- } 1 \text { combination } \\
\text { therapy }\end{array}$ & $\begin{array}{l}\text { Anti-PD-1 } \\
\text { monotherapy }\end{array}$ & & & Hazard Ratio (95\% Cl) & $P$ value \\
\hline \multicolumn{8}{|c|}{$0.47(0.20-1.10)$} \\
\hline $260 \mathrm{yr}$ & 13 & 8 & 5 & - & $H_{1}$ & $0.22(0.03-1.39)$ & 0.106 \\
\hline$<60 \mathrm{yr}$ & 24 & 14 & 10 & $\mapsto$ & $\longrightarrow$ & $0.63(0.23-1.70)$ & 0.358 \\
\hline \multicolumn{4}{|c|}{$0.00(0.20-1.10)$} & $\Leftrightarrow-1$ & 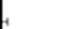 & $0.33(0.09-1.17)$ & 0.085 \\
\hline Male & 19 & 12 & 7 & $\ldots$ & $\square$ & $0.56(0.16-2.00)$ & 0.372 \\
\hline \multicolumn{8}{|l|}{ Smoking status } \\
\hline Current ot former & 12 & 6 & 6 & $\mapsto$ & 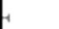 & $0.85(0.17-4.32)$ & 0.844 \\
\hline Never & 25 & 16 & 9 & $=-1$ & & $0.37(0.13-1.03)$ & 0.056 \\
\hline Overweight & 11 & 5 & 6 & $=1$ & 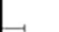 & 0.34 (0 08-1.53) & 0160 \\
\hline $\begin{array}{c}\text { Normal } \\
\text { ECOG }\end{array}$ & 26 & 17 & 9 & $\mapsto$ & $\longrightarrow$ & $0.61(0.21-1.80)$ & 0.369 \\
\hline \multicolumn{8}{|l|}{ ECOG performance status } \\
\hline 0.1 & 29 & 18 & 11 & $\mapsto=1$ & $\longrightarrow$ & $0.57(0.20-1.63)$ & 0.295 \\
\hline \multicolumn{8}{|l|}{ Surgery } \\
\hline Yes & 26 & 16 & 10 & $=-1$ & & $0.17(0.06-0.55)$ & 0.003 \\
\hline No & 11 & 6 & 5 & $\mapsto$ & & $2.42(0.46-12.63)$ & 0.295 \\
\hline \multicolumn{8}{|l|}{ Virus infection } \\
\hline Yes & 11 & 7 & 4 & $\mapsto$ & & $2.45(0.46-13.00)$ & 0.294 \\
\hline \multirow{2}{*}{\multicolumn{8}{|c|}{ Histological grade }} \\
\hline & & & & & & & \\
\hline Well + moderate & 16 & 8 & 8 & $\because 1$ & $\mathrm{~F}$ & $0.29(0.07-1.18)$ & 0.083 \\
\hline Poor + unknown & 21 & 14 & 7 & $=$ & & $0.63(0.19-2.05)$ & 0.441 \\
\hline \multicolumn{8}{|l|}{ First-line treatment } \\
\hline Yes & 19 & 10 & 9 & -1 & $\longrightarrow$ & $0.43(0.10-1.78)$ & 0.244 \\
\hline No & 18 & 12 & 6 & $\mapsto-1$ & H & $0.48(0.16-1.38)$ & 0.170 \\
\hline \multicolumn{8}{|l|}{ Liver metastases } \\
\hline Yes & 31 & 18 & 13 & $\pitchfork-$ & & $0.46(0.18-1.13)$ & 0.091 \\
\hline \multirow{2}{*}{\multicolumn{8}{|c|}{ Metastases number }} \\
\hline & & & & & & & \\
\hline$\geq 3$ & 13 & 10 & 3 & . & & ONA & 0.578 \\
\hline 0.2 & 24 & 12 & 12 & $\ldots$ & $\rightarrow$ & $0.44(0.13-1.47)$ & 0.182 \\
\hline \multirow{4}{*}{$\begin{array}{l}\text { Anti-PD-1 inhibitor } \\
\text { Pembrolizumab } \\
\text { Nivolumab }\end{array}$} & & & & & & & \\
\hline & 8 & 5 & 3 & $\mapsto-$ & & $0.58(0.09-3.58)$ & 0.558 \\
\hline & 29 & 17 & 12 & $\mapsto 1$ & & $0.46(0.17-1.21)$ & 0.116 \\
\hline & & & & 0 & 2 & 6 & \\
\hline
\end{tabular}

B

\begin{tabular}{|c|c|c|c|c|c|c|c|c|}
\hline Subgroup & $\begin{array}{l}\text { No. of } \\
\text { Patients }\end{array}$ & $\begin{array}{c}\text { Anti-PD- } 1 \text { combination } \\
\text { therapy }\end{array}$ & $\begin{array}{l}\text { Anti-PD-1 } \\
\text { monotherap! }\end{array}$ & & & & Hazard Ratio $(95 \% \mathrm{Cl})$ & $P$ value \\
\hline \multicolumn{8}{|l|}{ Age } & 0.134 \\
\hline$\geq 60 \mathrm{yr}$ & 13 & 8 & 5 & $\mapsto$ & 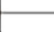 & & $0.83(0.21-3.33)$ & 0.787 \\
\hline$<60 \mathrm{yr}$ & 24 & 14 & 10 & 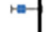 & & & $0.44(0.18-1.06)$ & 0.068 \\
\hline \multicolumn{9}{|l|}{ Sex } \\
\hline Female & 18 & 10 & 8 & 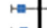 & H & & $0.47(0.16-1.34)$ & 0.157 \\
\hline Male & 19 & 12 & 7 & $\mapsto$ & $\longrightarrow$ & & $0.52(0.18-1.57)$ & 0.248 \\
\hline \multicolumn{9}{|l|}{ Smoking status } \\
\hline Current ot former & 12 & 6 & 6 & 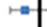 & $\longrightarrow$ & & $0.55(0.14-2.11)$ & 0.383 \\
\hline Never & 25 & 16 & 9 & $\cdots-$ & & & $0.44(0.18-1.09)$ & 0.075 \\
\hline \multicolumn{9}{|c|}{ ) } \\
\hline Overweight & 11 & 5 & 6 & $\mapsto-$ & & & $0.68(0.18-2.55)$ & 0.565 \\
\hline Normal & 26 & 17 & 9 & $=-1$ & 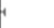 & & $0.47(0.19-1.17)$ & 0.104 \\
\hline \multicolumn{9}{|l|}{ ECOG performance status } \\
\hline$\geq 2$ & 8 & 4 & 4 & $=-$ & -1 & & $0.15(0.02-1.34)$ & 0.089 \\
\hline 0.1 & 29 & 18 & 11 & $1-0$ & $\multimap$ & & $0.72(0.31-1.64)$ & 0.430 \\
\hline \multicolumn{9}{|l|}{ Surgery } \\
\hline Yes & 26 & 16 & 10 & $=-1$ & & & $0.24(0.09-0.65)$ & 0.005 \\
\hline No & 11 & 6 & 5 & $\mapsto$ & 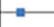 & & $1.61(0.44-5.89)$ & 0.472 \\
\hline \multicolumn{9}{|l|}{ Virus infection } \\
\hline Yes & 11 & 7 & 4 & r- & & & $0.73(0.19-2.77)$ & 0.648 \\
\hline No & 26 & 15 & 11 & $=-1$ & & & $0.42(0.17-1.04)$ & 0.061 \\
\hline \multicolumn{9}{|l|}{ Histological grade } \\
\hline Well + moderate & 16 & 8 & 8 & $=-1$ & & & $0.34(0.10-1.17)$ & 0.086 \\
\hline Poor + unknown & 21 & 14 & 7 & $\mapsto$ & $\longrightarrow$ & & $0.74(0.28-2.00)$ & 0.554 \\
\hline \multicolumn{9}{|l|}{ First-line treatment } \\
\hline Yes & 19 & 10 & 9 & $1=$ & $\longrightarrow$ & & $0.65(0.21-2.01)$ & 0.456 \\
\hline No & 18 & 12 & 6 & $=-1$ & 4 & & $0.44(0.16-1.26)$ & 0.128 \\
\hline \multicolumn{9}{|l|}{ Liver metastases } \\
\hline Yes & 31 & 18 & 13 & $r=1$ & - & & $0.60(0.27-1.33)$ & 0.212 \\
\hline No & 6 & 4 & 2 & $1=-$ & & & $0.38(0.05-2.76)$ & 0.339 \\
\hline \multicolumn{9}{|l|}{ Metastases number } \\
\hline$\geq 3$ & 13 & 10 & 3 & $=-1$ & & & $0.22(0.04-1.10)$ & 0.064 \\
\hline 0.2 & 24 & 12 & 12 & $=-$ & 4 & & $0.46(0.18-1.19)$ & 0.109 \\
\hline \multicolumn{9}{|l|}{ Anti-PD-1 inhibitor } \\
\hline Pembrolizumab & 8 & 5 & 3 & $\mapsto$ & $=-$ & & $1.61(0.31-8.47)$ & 0.571 \\
\hline \multirow[t]{2}{*}{ Nivolumab } & 29 & 17 & 12 & -1 & & & $0.52(0.22-1.21)$ & 0.127 \\
\hline & & & & 0 & 2 & 4 & 6 & \\
\hline
\end{tabular}

Figure S1 Treatment effect on overall survival (A) and progression-free survival (B) according to subgroup. 

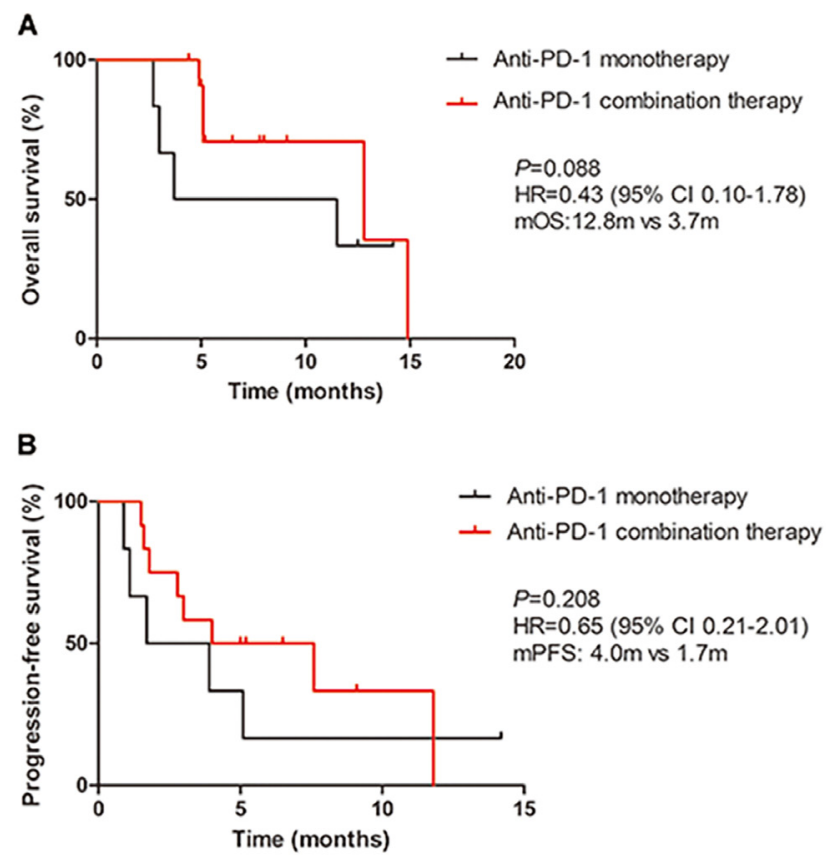

Figure S2 Kaplan-Meier estimates of overall survival and progression-free survival of patients as first-line treatment. (A) Kaplan-Meier survival curves of overall survival comparing anti-PD-1 monotherapy and combination therapy as the first-line treatment. (B) Kaplan-Meier survival curves of progression-free survival comparing anti-PD-1 monotherapy and combination therapy as the first-line treatment.
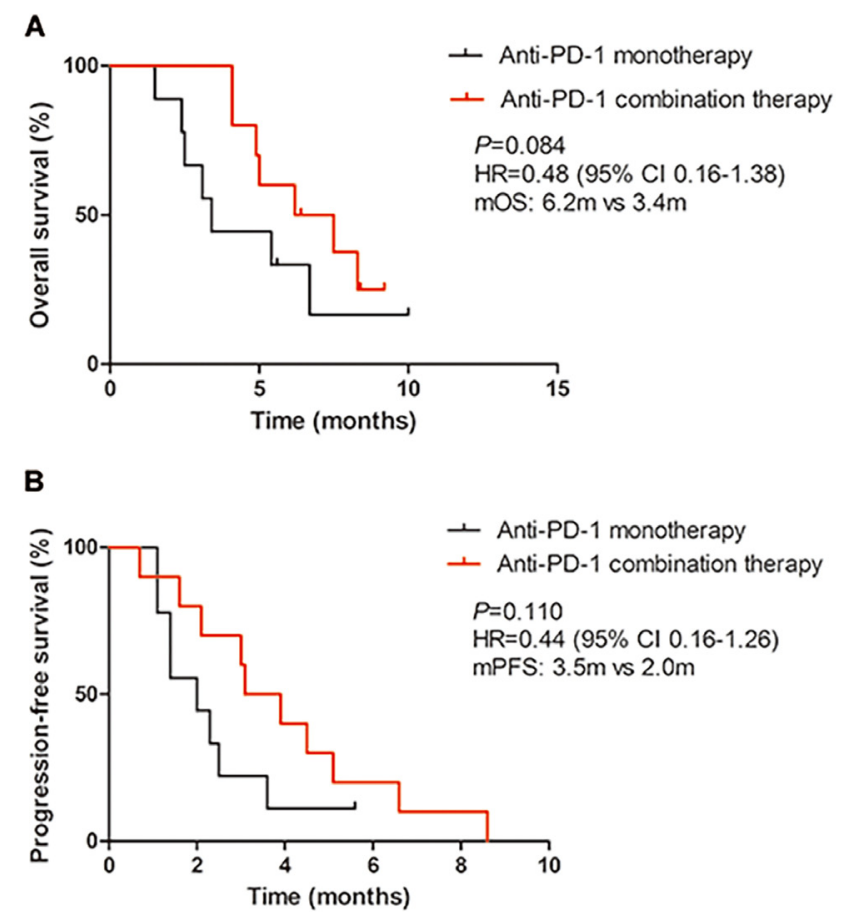

Figure S3 Kaplan-Meier estimates of overall survival and progression-free survival of patients as second-line treatment or beyond. (A) Kaplan-Meier survival curves of overall survival comparing anti-PD-1 monotherapy and combination therapy as the second-line treatment or beyond. (B) Kaplan-Meier survival curves of progression-free survival comparing anti-PD-1 monotherapy and combination therapy as the second-line treatment or beyond. 